\title{
Exponential energy decay and blow-up of solutions for a system of nonlinear viscoelastic wave equations with strong damping
}

Fei Liang ${ }^{1,2}$ and Hongjun Gao ${ }^{1 *}$

\footnotetext{
* Correspondence: gaohj@hotmail. com

${ }^{1}$ Jiangsu Provincial Key Laboratory for Numerical Simulation of Large Scale Complex Systems, School of Mathematical Sciences, Nanjing Normal University, Nanjing 210046, PR China

Full list of author information is available at the end of the article
}

\section{Abstract}

In this paper, we consider the system of nonlinear viscoelastic equations

$$
\begin{cases}u_{t t}-\Delta u+\int_{0}^{t} g_{1}(t-\tau) \Delta u(\tau) d \tau-\Delta u_{t}=f_{1}(u, v), & (x, t) \in \Omega \times(0, T), \\ v_{t t}-\Delta v+\int_{0}^{t} g_{2}(t-\tau) \Delta v(\tau) d \tau-\Delta v_{t}=f_{2}(u, v), & (x, t) \in \Omega \times(0, T)\end{cases}
$$

with initial and Dirichlet boundary conditions. We prove that, under suitable assumptions on the functions $g_{i}, f_{i}(i=1,2)$ and certain initial data in the stable set, the decay rate of the solution energy is exponential. Conversely, for certain initial data in the unstable set, there are solutions with positive initial energy that blow up in finite time.

2000 Mathematics Subject Classifications: 35L05; 35L55; 35L70.

Keywords: decay, blow-up, positive initial energy, viscoelastic wave equations

\section{Introduction}

In this article, we study the following system of viscoelastic equations:

$$
\begin{cases}u_{t t}-\Delta u+\int_{0}^{t} g_{1}(t-\tau) \Delta u(\tau) d \tau-\Delta u_{t}=f_{1}(u, v), & (x, t) \in \Omega \times(0, T), \\ v_{t t}-\Delta v+\int_{0}^{t} g_{2}(t-\tau) \Delta v(\tau) d \tau-\Delta v_{t}=f_{2}(u, v), & (x, t) \in \Omega \times(0, T), \\ u(x, t)=v(x, t)=0, & x \in \partial \Omega \times(0, T), \\ u(x, 0)=u_{0}(x), \quad u_{t}(x, 0)=u_{1}(x), & x \in \Omega, \\ v(x, 0)=v_{0}(x), \quad v_{t}(x, 0)=v_{1}(x), & x \in \Omega,\end{cases}
$$

where $\Omega$ is a bounded domain in $\mathbb{R}^{n}$ with a smooth boundary $\partial \Omega$, and $g_{i}(\cdot): \mathbb{R}_{+} \rightarrow \mathbb{R}$ ${ }_{+}, f_{i}(\cdot, \cdot): \mathbb{R}^{2} \rightarrow \mathbb{R}(i=1,2)$ are given functions to be specified later. Here, $u$ and $v$ denote the transverse displacements of waves. This problem arises in the theory of viscoelastic and describes the interaction of two scalar fields, we can refer to Cavalcanti et al. [1], Messaoudi and Tatar [2], Renardy et al. [3].

To motivate this study, let us recall some results regarding single viscoelastic wave equation. Cavalcanti et al. [4] studied the following equation:

$$
u_{t t}-\Delta u+\int_{0}^{t} g(t-\tau) \Delta u(\tau) d \tau+a(x) u_{t}+|u|^{\gamma} u=0, \quad \text { in } \Omega \times(0, \infty)
$$

(c) 2011 fei and Hongjun; licensee Springer. This is an Open Access article distributed under the terms of the Creative Commons Attribution License (http://creativecommons.org/licenses/by/2.0), which permits unrestricted use, distribution, and reproduction in any medium, provided the original work is properly cited. 
for $a: \Omega \rightarrow \mathbb{R}^{+}$, a function, which may be null on a part of the domain $\Omega$. Under the conditions that $a(x) \geq a_{0}>0$ on $\Omega_{1} \subset \Omega$, with $\Omega_{1}$ satisfying some geometry restrictions and

$$
-\xi_{1} g(t) \leq g^{\prime}(t) \leq-\xi_{2} g(t), \quad t \geq 0,
$$

the authors established an exponential rate of decay. This latter result has been improved by Cavalcanti and Oquendo [5] and Berrimi and Messaoudi [6]. In their study, Cavalcanti and Oquendo [5] considered the situation where the internal dissipation acts on a part of $\Omega$ and the viscoelastic dissipation acts on the other part. They established both exponential and polynomial decay results under the conditions on $g$ and its derivatives up to the third order, whereas Berrimi and Messaoudi [6] allowed the internal dissipation to be nonlinear. They also showed that the dissipation induced by the integral term is strong enough to stabilize the system and established an exponential decay for the solution energy provided that $g$ satisfies a relation of the form

$$
g^{\prime}(t) \leq-\xi g(t), \quad t \geq 0 .
$$

Cavalcanti et al. [1] also studied, in a bounded domain, the following equation:

$$
\left|u_{t}\right|^{\rho} u_{t t}-\Delta u-\Delta u_{t t}+\int_{0}^{t} g_{1}(t-\tau) \Delta u(\tau) d \tau-\gamma \Delta u_{t}=0
$$

$\rho>0$, and proved a global existence result for $\gamma \geq 0$ and an exponential decay for $\gamma>$ 0 . This result has been extended by Messaoudi and Tatar [2,7] to the situation where $\gamma$ $=0$ and exponential and polynomial decay results in the absence, as well as in the presence, of a source term have been established. Recently, Messaoudi [8,9] considered

$$
u_{t t}-\Delta u+\int_{0}^{t} g_{1}(t-\tau) \Delta u(\tau) d \tau=b|u|^{\gamma} u, \quad(x, t) \in \Omega \times(0, \infty)
$$

for $b=0$ and $b=1$ and for a wider class of relaxation functions. He established a more general decay result, for which the usual exponential and polynomial decay results are just special cases.

For the finite time blow-up of a solution, the single viscoelastic wave equation of the form

$$
u_{t t}-\Delta u+\int_{0}^{t} g(t-\tau) \Delta u(\tau) d \tau+h\left(u_{t}\right)=f(u)
$$

in $\Omega \times(0, \infty)$ with initial and boundary conditions has extensively been studied. See in this regard, Kafini and Messaoudi [10], Messaoudi [11,12], Song and Zhong [13], Wang [14]. For instance, Messaoudi [11] studied (1.2) for $h\left(u_{t}\right)=a\left|u_{t}\right|^{m-2} u_{t}$ and $f(u)=$ $b|u|^{p-2} u$ and proved a blow-up result for solutions with negative initial energy if $p>m$ $\geq 2$ and a global result for $2 \leq p \leq m$. This result has been later improved by Messaoudi [12] to accommodate certain solutions with positive initial energy. Song and Zhong [13] considered (1.2) for $h\left(u_{t}\right)=-\Delta u_{t}$ and $f(u)=|u|^{p-2} u$ and proved a blow-up result for solutions with positive initial energy using the ideas of the "potential well" theory introduced by Payne and Sattinger [15]. 
This study is also motivated by the research of the well-known Klein-Gordon system

$$
\left\{\begin{array}{l}
u_{t t}-\Delta u+m_{1} u+k_{1} u v^{2}=0 \\
v_{t t}-\Delta v+m_{2} v+k_{2} u^{2} v=0
\end{array}\right.
$$

which arises in the study of quantum field theory [16]. See also Medeiros and Miranda [17], Zhang [18] for some generalizations of this system and references therein. As far as we know, the problem (1.1) with the viscoelastic effect described by the memory terms has not been well studied. Recently, Han and Wang [19] considered the following problem

$$
\begin{cases}u_{t t}-\Delta u+\int_{0}^{t} g_{1}(t-\tau) \Delta u(\tau) d \tau+\left|u_{t}\right|^{m-1} u_{t}=f_{1}(u, v), & (x, t) \in \Omega \times(0, T), \\ v_{t t}-\Delta v+\int_{0}^{t} g_{2}(t-\tau) \Delta v(\tau) d \tau+\left|v_{t}\right|^{r-1} v_{t}=f_{2}(u, v), & (x, t) \in \Omega \times(0, T), \\ u(x, t)=v(x, t)=0, & x \in \partial \Omega \times(0, T), \\ u(x, 0)=u_{0}(x), \quad u_{t}(x, 0)=u_{1}(x), & x \in \Omega, \\ v(x, 0)=v_{0}(x), \quad v_{t}(x, 0)=v_{1}(x), & x \in \Omega,\end{cases}
$$

where $\Omega$ is a bounded domain with smooth boundary $\partial \Omega$ in $\mathbb{R}^{n}, n=1,2,3$. Under suitable assumptions on the functions $g_{i}, f_{i}(i=1,2)$, the initial data and the parameters in the equations, they established several results concerning local existence, global existence, uniqueness, and finite time blow-up (the initial energy $E(0)<0$ ) property. This latter blow-up result has been improved by Messaoudi and Said-Houari [20], to certain solutions with positive initial energy. Liu [21] studied the following system

$$
\begin{cases}\left|u_{t}\right|^{\rho} u_{t t}-\Delta u-\gamma_{1} \Delta u_{t t}+\int_{0}^{t} g_{1}(t-\tau) \Delta u(\tau) d \tau+f(u, v)=0, & (x, t) \in \Omega \times(0, T) \\ \left|v_{t}\right|^{\rho} v_{t t}-\Delta v-\gamma_{2} \Delta v_{t t}+\int_{0}^{t} g_{2}(t-\tau) \Delta v(\tau) d \tau+k(u, v)=0, & (x, t) \in \Omega \times(0, T) \\ u(x, t)=v(x, t)=0, & x \in \partial \Omega \times(0, T) \\ u(x, 0)=u_{0}(x), \quad u_{t}(x, 0)=u_{1}(x), & x \in \Omega, \\ v(x, 0)=v_{0}(x), \quad v_{t}(x, 0)=v_{1}(x), & x \in \Omega,\end{cases}
$$

where $\Omega$ is a bounded domain with smooth boundary $\partial \Omega$ in $\mathbb{R}^{n}, \gamma_{1}, \gamma_{2} \geq 0$ are constants and $\rho$ is a real number such that $0<\rho \leq 2 /(n-2)$ if $n \geq 3$ or $\rho>0$ if $n=1,2$. Under suitable assumptions on the functions $g(s), h(s), f(u, v), k(u, v)$, they used the perturbed energy method to show that the dissipations given by the viscoelastic terms are strong enough to ensure exponential or polynomial decay of the solutions energy, depending on the decay rate of the relaxation functions $g(s)$ and $h(s)$. For the problem (1.1) in $\mathbb{R}^{n}$, we mention the work of Kafini and Messaoudi [10].

Motivated by the above research, we consider in this study the coupled system (1.1). We prove that, under suitable assumptions on the functions $g_{i}, f_{i}(i=1,2)$ and certain initial data in the stable set, the decay rate of the solution energy is exponential. Conversely, for certain initial data in the unstable set, there are solutions with positive initial energy that blow up in finite time.

This article is organized as follows. In Section 2, we present some assumptions and definitions needed for this study. Section 3 is devoted to the proof of the uniform decay result. In Section 4, we prove the blow-up result.

\section{Preliminaries}

First, let us introduce some notation used throughout this article. We denote by $\|\cdot\|_{q}$ the $L^{q}(\Omega)$ norm for $1 \leq q \leq \infty$ and by $\|\nabla \cdot\|_{2}$ the Dirichlet norm in $H_{0}^{1}(\Omega)$ which is 
equivalent to the $H^{1}(\Omega)$ norm. Moreover, we set

$$
(\varphi, \psi)=\int_{\Omega} \varphi(x) \psi(x) d x
$$

as the usual $L^{2}(\Omega)$ inner product.

Concerning the functions $f_{1}(u, v)$ and $f_{2}(u, v)$, we take

$$
\begin{aligned}
& f_{1}(u, v)=\left[a|u+v|^{2(p+1)}(u+v)+b|u|^{p} u|v|^{(p+2)}\right], \\
& f_{2}(u, v)=\left[a|u+v|^{2(p+1)}(u+v)+b|u|^{(p+2)}|v|^{p} v\right],
\end{aligned}
$$

where $a, b>0$ are constants and $p$ satisfies

$$
\begin{cases}p>-1, & \text { if } n=1,2 \\ -1<p \leq 1, & \text { if } n=3\end{cases}
$$

One can easily verify that

$$
u f_{1}(u, v)+v f_{2}(u, v)=2(p+2) F(u, v), \quad \forall(u, v) \in \mathbb{R}^{2},
$$

where

$$
F(u, v)=\frac{1}{2(p+2)}\left[a|u+v|^{2(p+2)}+2 b|u v|^{p+2}\right] .
$$

For the relaxation functions $g_{i}(t)(i=1,2)$, we assume

(G1) $g_{i}(t): \mathbb{R}_{+} \rightarrow \mathbb{R}_{+}$belong to $C^{1}\left(\mathbb{R}_{+}\right)$and satisfy

$$
g_{i}(t) \geq 0, \quad g_{i}^{\prime}(t) \leq 0, \quad \text { for } t \geq 0
$$

and

$$
1-\int_{0}^{\infty} g_{i}(s) d s=k_{i}>0 .
$$

(G2) $\max \left\{\int_{0}^{\infty} g_{1}(s) d s, \int_{0}^{\infty} g_{2}(s) d s\right\}<\frac{4(p+1)(p+2)}{4(p+1)(p+2)+1}$.

We next state the local existence and the uniqueness of the solution of problem (1.1), whose proof can be found in Han and Wang [19] (Theorem 2.1) with slight modification, so we will omit its proof. In the proof, the authors adopted the technique of Agre and Rammaha [22] which consists of constructing approximations by the Faedo-Galerkin procedure without imposing the usual smallness conditions on the initial data to handle the source terms. Unfortunately, due to the strong nonlinearities on $f_{1}$ and $f_{2}$, the techniques used by Han and Wang [19] and Agre and Rammaha [22] allowed them to prove the local existence result only for $n \leq 3$. We note that the local existence result in the case of $n>3$ is still open. For related results, we also refer the reader to Said-Houari and Messaoudi [23] and Messaoudi and Said-Houari [20]. So throughout this article, we have assumed that $n \leq 3$.

Theorem 2.1. Assume that (2.1) and (G1) hold, and that $\left(u_{0}, u_{1}\right) \in H_{0}^{1}(\Omega) \times L^{2}(\Omega)$, $\left(v_{0}, v_{1}\right) \in H_{0}^{1}(\Omega) \times L^{2}(\Omega)$. Then problem (1.1) has a unique local solution

$$
u, v \in C\left([0, T) ; H_{0}^{1}(\Omega)\right), \quad u_{t}, v_{t} \in C\left([0, T) ; L^{2}(\Omega)\right) \cap L^{2}\left([0, T) ; H_{0}^{1}(\Omega)\right)
$$


for some $T>0$. If $T<\infty$, then

$$
\lim _{t \rightarrow T}\left(k_{1}\|\nabla u(t)\|_{2}^{2}+\left\|u_{t}(t)\right\|_{2}^{2}+k_{2}\|\nabla v(t)\|_{2}^{2}+\left\|v_{t}(t)\right\|_{2}^{2}\right)=\infty .
$$

Finally, we define

$$
\begin{aligned}
I(t)= & \left(1-\int_{0}^{t} g_{1}(\tau) d \tau\right)\|\nabla u(t)\|_{2}^{2}+\left(1-\int_{0}^{t} g_{2}(\tau) d \tau\right)\|\nabla v(t)\|_{2}^{2} \\
& +\left[\left(g_{1} \circ \nabla u\right)(t)+\left(g_{2} \circ \nabla v\right)(t)\right]-2(p+2) \int_{\Omega} F(u, v) d x, \\
J(t)= & \frac{1}{2}\left[\left(1-\int_{0}^{t} g_{1}(\tau) d \tau\right)\|\nabla u(t)\|_{2}^{2}+\left(1-\int_{0}^{t} g_{2}(\tau) d \tau\right)\|\nabla v(t)\|_{2}^{2}\right] \\
+ & \frac{1}{2}\left[\left(g_{1} \circ \nabla u\right)(t)+\left(g_{2} \circ \nabla v\right)(t)\right]-\int_{\Omega} F(u, v) d x,
\end{aligned}
$$

such functionals we could refer to Muñoz Rivera [24,25]. We also define the energy function as follows

$$
E(t)=\frac{1}{2}\left(\left\|u_{t}(t)\right\|_{2}^{2}+\left\|v_{t}(t)\right\|_{2}^{2}\right)+J(t),
$$

where

$$
\left(g_{i} \circ w\right)(t)=\int_{0}^{t} g_{i}(t-\tau)\|w(t)-w(\tau)\|_{2}^{2} d \tau .
$$

\section{Global existence and energy decay}

In this section, we deal with the uniform exponential decay of the energy for system (1.1) by using the perturbed energy method. Before we state and prove our main result, we need the following lemmas.

Lemma 3.1. Assume (2.1) and (G1) hold. Let $(u, v)$ be the solution of the system (1.1), then the energy functional is a decreasing function, that is

$$
\begin{aligned}
E^{\prime}(t)= & -\left\|\nabla u_{t}(t)\right\|_{2}^{2}-\left\|\nabla v_{t}(t)\right\|_{2}^{2}+\frac{1}{2}\left(g_{1}^{\prime} \circ u\right)(t)+\frac{1}{2}\left(g_{2}^{\prime} \circ v\right)(t) \\
& -\frac{1}{2} g_{1}(t)\|\nabla u(t)\|_{2}^{2}-\frac{1}{2} g_{2}(t)\|\nabla v(t)\|_{2}^{2} \leq 0 .
\end{aligned}
$$

Moreover, the following energy inequality holds:

$$
E(t)+\int_{s}^{t}\left(\left\|\nabla u_{t}(\tau)\right\|_{2}^{2}+\left\|\nabla v_{t}(\tau)\right\|_{2}^{2}\right) d \tau \leq E(s), \quad \text { for } 0 \leq s \leq t<T .
$$

Lemma 3.2. Let (2.1) hold. Then, there exists $\eta>0$ such that for any $(u, v) \in H_{0}^{1}(\Omega) \times H_{0}^{1}(\Omega)$, we have

$$
\|u+v\|_{2(p+2)}^{2(p+2)}+2\|u v\|_{p+2}^{p+2} \leq \eta\left(k_{1}\|\nabla u(t)\|_{2}^{2}+k_{2}\|\nabla v(t)\|_{2}^{2}\right)^{p+2} .
$$

Proof. The proof is almost the same that of Said-Houari [26], so we omit it here. To prove our result and for the sake of simplicity, we take $a=b=1$ and introduce the following: 


$$
B=\eta^{\frac{1}{2(p+2)}}, \quad \alpha^{*}=B^{-\frac{p+2}{P+1}}, \quad E_{1}=\left(\frac{1}{2}-\frac{1}{2(p+2)}\right) \alpha^{* 2},
$$

where $\eta$ is the optimal constant in (3.3). The following lemma will play an essential role in the proof of our main result, and it is similar to a lemma used first by Vitillaro [27], to study a class of a single wave equation, which introduces a potential well.

Lemma 3.3. Let (2.1) and (G1) hold. Let (u,v) be the solution of the system (1.1). Assume further that $E(0)<E_{1}$ and

$$
\left(k_{1}\left\|\nabla u_{0}\right\|_{2}^{2}+k_{2}\left\|\nabla v_{0}\right\|_{2}^{2}\right)^{1 / 2}<\alpha^{*},
$$

Then

$$
\left(k_{1}\|\nabla u(t)\|_{2}^{2}+k_{2}\|\nabla v(t)\|_{2}^{2}+\left(g_{1} \circ \nabla u\right)(t)+\left(g_{2} \circ \nabla v\right)(t)\right)^{1 / 2}<\alpha^{*}, \quad \text { for } t \in[0, T) .
$$

Proof. We first note that, by (2.5), (3.3) and the definition of $B$, we have

$$
\begin{aligned}
E(t) & \geq \frac{1}{2}\left(k_{1}\|\nabla u(t)\|_{2}^{2}+k_{2}\|\nabla v(t)\|_{2}^{2}+\left(g_{1} \circ \nabla u\right)(t)+\left(g_{2} \circ \nabla v\right)(t)\right) \\
& -\frac{1}{2(p+2)}\left(\|u+v\|_{2(p+2)}^{2(p+2)}+2\|u v\|_{p+2}^{p+2}\right) \\
& \geq \frac{1}{2}\left(k_{1}\|\nabla u(t)\|_{2}^{2}+k_{2}\|\nabla v(t)\|_{2}^{2}+\left(g_{1} \circ \nabla u\right)(t)+\left(g_{2} \circ \nabla v\right)(t)\right) \\
& -\frac{B^{2(p+2)}}{2(p+2)}\left(k_{1}\|\nabla u(t)\|_{2}^{2}+k_{2}\|\nabla v(t)\|_{2}^{2}\right)^{p+2} \\
& \geq \frac{1}{2} \alpha^{2}-\frac{B^{2(p+2)}}{2(p+2)} \alpha^{2(p+2)}=g(\alpha),
\end{aligned}
$$

where $\alpha=\left(k_{1}\|\nabla u(t)\|_{2}^{2}+k_{2}\|\nabla v(t)\|_{2}^{2}+\left(g_{1} \circ \nabla u\right)(t)+\left(g_{2} \circ \nabla v\right)(t)\right)^{1 / 2}$. It is not hard to verify that $g$ is increasing for $0<\alpha<\alpha^{*}$, decreasing for $\alpha>\alpha^{*}, g(\alpha) \rightarrow-\infty$ as $\alpha \rightarrow$ $+\infty$, and

$$
g\left(\alpha^{*}\right)=\frac{1}{2} \alpha^{* 2}-\frac{B^{2(p+2)}}{2(p+2)} \alpha^{* 2(p+2)}=E_{1}
$$

where $\alpha^{*}$ is given in (3.4). Now we establish (3.6) by contradiction. Suppose (3.6) does not hold, then it follows from the continuity of $(u(t), v(t))$ that there exists $t_{0} \in$ $(0, T)$ such that

$$
\left(k_{1}\left\|\nabla u\left(t_{0}\right)\right\|_{2}^{2}+k_{2}\left\|\nabla v\left(t_{0}\right)\right\|_{2}^{2}+\left(g_{1} \circ \nabla u\right)\left(t_{0}\right)+\left(g_{2} \circ \nabla v\right)\left(t_{0}\right)\right)^{1 / 2}=\alpha^{*} .
$$

By (3.7), we observe that

$$
E\left(t_{0}\right) \geq g\left(\left(k_{1}\left\|\nabla u\left(t_{0}\right)\right\|_{2}^{2}+k_{2}\left\|\nabla v\left(t_{0}\right)\right\|_{2}^{2}+\left(g_{1} \circ \nabla u\right)\left(t_{0}\right)+\left(g_{2} \circ \nabla v\right)\left(t_{0}\right)\right)^{1 / 2}\right)=g\left(\alpha^{*}\right)=E_{1} .
$$

This is impossible since $E(t) \leq E(0)<E_{1}$ for all $t \in[0, T)$. Hence (3.6) is established. $\square$ The following integral inequality plays an important role in our proof of the energy decay of the solutions to problem (1.1).

Lemma 3.4. [28] Assume that the function $\phi: \mathbb{R}^{+} \cup\{0\} \rightarrow \mathbb{R}^{+} \cup\{0\}$ is a non-increasing function and that there exists a constant $c>0$ such that

$$
\int_{t}^{\infty} \varphi(s) d s \leq c \varphi(t)
$$


for every $t \in[0, \infty)$. Then

$$
\varphi(t) \leq \varphi(0) \exp (1-t / c)
$$

for every $t \geq c$.

Theorem 3.5. Let (2.1) and (G1) hold. If the initial data $\left(u_{0}, u_{1}\right) \in H_{0}^{1}(\Omega) \times L^{2}(\Omega)$, $\left(v_{0}, v_{1}\right) \in H_{0}^{1}(\Omega) \times L^{2}(\Omega)$ satisfy $E(0)<E_{1}$ and

$$
\left(k_{1}\left\|\nabla u_{0}\right\|_{2}^{2}+k_{2}\left\|\nabla v_{0}\right\|_{2}^{2}\right)^{1 / 2}<\alpha^{*},
$$

where the constants $\alpha_{*}, E_{1}$ are defined in (3.4), then the corresponding solution to (1.1) globally exists, i.e. $T=\infty$. Moreover, if the initial energy $E(0)$ and $k$ such that

$$
1-\eta\left(\frac{2(p+2)}{(p+1)} E(0)\right)^{(p+1)}-\frac{5(1-k)(p+2)}{2 k(p+1)}>0,
$$

where $k=\min \left\{k_{1}, k_{2}\right\}$, then the energy decay is

$$
E(t) \leq E(0) \exp \left(1-a C^{-1} t\right)
$$

for every $t \geq a C^{-1}$, where $C$ is some positive constant.

Proof. In order to get $T=\infty$, by (2.2), it suffices to show that

$$
\left\|u_{t}(t)\right\|_{2}^{2}+\left\|v_{t}(t)\right\|_{2}^{2}+k_{1}\|\nabla u(t)\|_{2}^{2}+k_{2}\|\nabla v(t)\|_{2}^{2}
$$

is bounded independently of $t$. Since $E(0)<E_{1}$ and

$$
\left(k_{1}\left\|\nabla u_{0}\right\|_{2}^{2}+k_{2}\left\|\nabla v_{0}\right\|_{2}^{2}\right)^{1 / 2}<\alpha^{*},
$$

it follows from Lemma 3.3 that

$$
k_{1}\|\nabla u(t)\|_{2}^{2}+k_{2}\|\nabla v(t)\|_{2}^{2} \leq k_{1}\|\nabla u(t)\|_{2}^{2}+k_{2}\|\nabla v(t)\|_{2}^{2}+\left(g_{1} \circ \nabla u\right)(t)+\left(g_{2} \circ \nabla v\right)(t)<\alpha^{* 2},
$$

which implies that

$$
\begin{aligned}
I(t) & \geq k_{1}\|\nabla u(t)\|_{2}^{2}+k_{2}\|\nabla v(t)\|_{2}^{2}+\left[\left(g_{1} \circ \nabla u\right)(t)+\left(g_{2} \circ \nabla v\right)(t)\right]-2(p+2) \int_{\Omega} F(u, v) d x \\
& \geq k_{1}\|\nabla u(t)\|_{2}^{2}+k_{2}\|\nabla v(t)\|_{2}^{2}-2(p+2) \int_{\Omega} F(u, v) d x \\
& =k_{1}\|\nabla u(t)\|_{2}^{2}+k_{2}\|\nabla v(t)\|_{2}^{2}-\left(\|u+v\|_{2(p+2)}^{2(p+2)}+2\|u v\|_{p+2}^{p+2}\right) \\
& \geq k_{1}\|\nabla u(t)\|_{2}^{2}+k_{2}\|\nabla v(t)\|_{2}^{2}-\eta\left(k_{1}\|\nabla u(t)\|_{2}^{2}+k_{2}\|\nabla v(t)\|_{2}^{2}\right)^{p+2} \geq 0, \quad \text { for } t \in[0, T),
\end{aligned}
$$

where we have used (3.3). Furthermore, by (2.3) and (2.4), we get

$$
\begin{aligned}
J(t) & \geq\left(\frac{1}{2}-\frac{1}{2(p+2)}\right)\left[\left(1-\int_{0}^{t} g_{1}(s) d s\right)\|\nabla u(t)\|_{2}^{2}+\left(1-\int_{0}^{t} g_{2}(s) d s\right)\|\nabla v(t)\|_{2}^{2}\right] \\
& +\left(\frac{1}{2}-\frac{1}{2(p+2)}\right)\left[\left(g_{1} \circ \nabla u\right)(t)+\left(g_{2} \circ \nabla v\right)(t)\right]+\frac{1}{2(p+2)} I(t) \\
& \geq \frac{p+1}{2(p+2)}\left[k_{1}\|\nabla u(t)\|_{2}^{2}+k_{2}\|\nabla v(t)\|_{2}^{2}+\left(g_{1} \circ \nabla u\right)(t)+\left(g_{2} \circ \nabla v\right)(t)\right]+\frac{1}{2(p+2)} I(t) \geq 0,
\end{aligned}
$$

from which, the definition of $E(t)$ and $E(t) \leq E(0)$, we deduce that

$$
\left[k_{1}\|\nabla u(t)\|_{2}^{2}+k_{2}\|\nabla v(t)\|_{2}^{2}\right] \leq \frac{2(p+2)}{(p+1)} J(t) \leq \frac{2(p+2)}{(p+1)} E(t) \leq \frac{2(p+2)}{(p+1)} E(0),
$$


for $t \in[0, T)$. So it follows from (16) and Lemma 3.1 that

$$
\begin{aligned}
\frac{p+1}{2(p+2)}\left[k_{1}\|\nabla u(t)\|_{2}^{2}+k_{2}\|\nabla v(t)\|_{2}^{2}\right]+\frac{1}{2}\left(\left\|u_{t}(t)\right\|_{2}^{2}+\left\|v_{t}(t)\right\|_{2}^{2}\right) & \leq J(t)+\frac{1}{2}\left(\left\|u_{t}(t)\right\|_{2}^{2}+\left\|v_{t}(t)\right\|_{2}^{2}\right) \\
& =E(t) \leq E(0)<E_{1}, \forall t \in[0, T),
\end{aligned}
$$

which implies

$$
\left\|u_{t}(t)\right\|_{2}^{2}+\left\|v_{t}(t)\right\|_{2}^{2}+k_{1}\|\nabla u(t)\|_{2}^{2}+k_{2}\|\nabla v(t)\|_{2}^{2}<C E_{1},
$$

where $C$ is a positive constant depending only on $p$.

Next we want to derive the decay rate of energy function for problem (1.1). By multiplying the first equation of system (1.1) by $u$ and the second equation of system (1.1) by $v$, integrating over $\Omega \times\left[t_{1}, t_{2}\right]\left(0 \leq t_{1} \leq t_{2}\right)$, using integration by parts and summing up, we have

$$
\begin{aligned}
& \left.\int_{\Omega} u_{t}(t) u(t) d x\right|_{t_{1}} ^{t_{2}}-\int_{t_{1}}^{t_{2}}\left\|u_{t}(t)\right\|_{2}^{2} d t+\left.\int_{\Omega} v_{t}(t) v(t) d x\right|_{t_{1}} ^{t_{2}}-\int_{t_{1}}^{t_{2}}\left\|v_{t}(t)\right\|_{2}^{2} d t \\
= & -\int_{t_{1}}^{t_{2}}\left(\nabla u(t), \nabla u_{t}(t)\right) d t-\int_{t_{1}}^{t_{2}}\left(\nabla v(t), \nabla v_{t}(t)\right) d t-\int_{t_{1}}^{t_{2}}\|\nabla u(t)\|_{2}^{2} d t-\int_{t_{1}}^{t_{2}}\|\nabla v(t)\|_{2}^{2} d t \\
& -\int_{t_{1}}^{t_{2}} \int_{\Omega} \int_{0}^{t} g_{1}(t-\tau) \Delta u(\tau) d \tau u(t) d x d t-\int_{t_{1}}^{t_{2}} \int_{\Omega} \int_{0}^{t} g_{2}(t-\tau) \Delta v(\tau) d \tau v(t) d x d t \\
& +2(p+2) \int_{t_{1}}^{t_{2}} \int_{\Omega} F(u, v) d x d t,
\end{aligned}
$$

which implies

$$
\begin{aligned}
& 2 \int_{t_{1}}^{t_{2}} E(t) d t-2(p+1) \int_{t_{1}}^{t_{2}} \int_{\Omega} F(u, v) d x d t \\
= & -\left.\int_{\Omega} u_{t}(t) u(t) d x\right|_{t_{1}} ^{t_{2}}-\left.\int_{\Omega} v_{t}(t) v(t) d x\right|_{t_{1}} ^{t_{2}}+2 \int_{t_{1}}^{t_{2}}\left\|u_{t}(t)\right\|_{2}^{2} d t+2 \int_{t_{1}}^{t_{2}}\left\|v_{t}(t)\right\|_{2}^{2} d t \\
& +\int_{t_{1}}^{t_{2}}\left(g_{1} \circ \nabla u\right)(t) d t+\int_{t_{1}}^{t_{2}}\left(g_{2} \circ \nabla v\right)(t) d t-\int_{t_{1}}^{t_{2}} \int_{0}^{t} g_{1}(\tau) d \tau\|\nabla u(t)\|_{2}^{2} d t \\
& -\int_{t_{1}}^{t_{2}} \int_{0}^{t} g_{2}(\tau) d \tau\|\nabla v(t)\|_{2}^{2} d t-\int_{t_{1}}^{t_{2}}\left(\nabla u(t), \nabla u_{t}(t)\right) d t-\int_{t_{1}}^{t_{2}}\left(\nabla v(t), \nabla v_{t}(t)\right) d t \\
& -\int_{t_{1}}^{t_{2}} \int_{\Omega} \int_{0}^{t} g_{1}(t-\tau) \Delta u(\tau) d \tau u(t) d x d t-\int_{t_{1}}^{t_{2}} \int_{\Omega} \int_{0}^{t} g_{2}(t-\tau) \Delta v(\tau) d \tau v(t) d x d t .
\end{aligned}
$$

For the 11th term on the right-hand side of (3.10), one has

$$
\begin{aligned}
& -2 \int_{\Omega} \int_{0}^{t} g_{1}(t-\tau) \Delta u(\tau) d \tau u(t) d x=2 \int_{\Omega} \int_{0}^{t} g_{1}(t-\tau) \nabla u(\tau) \nabla u(t) d \tau d x \\
& =\int_{0}^{t} g_{1}(t-\tau)\left(\|\nabla u(t)\|_{2}^{2}+\|\nabla u(\tau)\|_{2}^{2}\right) d \tau-\int_{0}^{t} g_{1}(t-\tau)\left(\|\nabla u(t)-\nabla u(\tau)\|_{2}^{2}\right) d \tau .
\end{aligned}
$$

Similarly,

$$
\begin{aligned}
& -2 \int_{\Omega} \int_{0}^{t} g_{2}(t-\tau) \Delta v(\tau) d \tau v(t) d x \\
& =\int_{0}^{t} g_{2}(t-\tau)\left(\|\nabla v(t)\|_{2}^{2}+\|\nabla v(\tau)\|_{2}^{2}\right) d \tau-\int_{0}^{t} g_{2}(t-\tau)\left(\|\nabla v(t)-\nabla v(\tau)\|_{2}^{2}\right) d \tau .
\end{aligned}
$$


Combining (3.10), (3.11) with (3.12), we have

$$
\begin{aligned}
& 2 \int_{t_{1}}^{t_{2}} E(t) d t-2(p+1) \int_{t_{1}}^{t_{2}} \int_{\Omega} F(u, v) d x d t \\
= & -\left.\int_{\Omega} u_{t}(t) u(t) d x\right|_{t_{1}} ^{t_{2}}-\left.\int_{\Omega} v_{t}(t) v(t) d x\right|_{t_{1}} ^{t_{2}}+2 \int_{t_{1}}^{t_{2}}\left\|u_{t}(t)\right\|_{2}^{2} d t+2 \int_{t_{1}}^{t_{2}}\left\|v_{t}(t)\right\|_{2}^{2} d t \\
& +\frac{1}{2} \int_{t_{1}}^{t_{2}}\left(g_{1} \circ \nabla u\right)(t) d t+\frac{1}{2} \int_{t_{1}}^{t_{2}}\left(g_{2} \circ \nabla v\right)(t) d t-\frac{1}{2} \int_{t_{1}}^{t_{2}} \int_{0}^{t} g_{1}(\tau) d \tau\|\nabla u(t)\|_{2}^{2} d t \\
& -\frac{1}{2} \int_{t_{1}}^{t_{2}} \int_{0}^{t} g_{2}(\tau) d \tau\|\nabla v(t)\|_{2}^{2} d t-\int_{t_{1}}^{t_{2}}\left(\nabla u(t), \nabla u_{t}(t)\right) d t-\int_{t_{1}}^{t_{2}}\left(\nabla v(t), \nabla v_{t}(t)\right) d t \\
& +\frac{1}{2} \int_{t_{1}}^{t_{2}} \int_{0}^{t} g_{1}(t-\tau)\|\nabla u(\tau)\|_{2}^{2} d \tau d t+\frac{1}{2} \int_{t_{1}}^{t_{2}} \int_{0}^{t} g_{2}(t-\tau)\|\nabla v(\tau)\|_{2}^{2} d \tau d t \\
\leq & -\left.\int_{\Omega}^{u_{t}} u_{t}(t) u(t) d x\right|_{t_{1}} ^{t_{2}}-\left.\int_{\Omega} v_{t}(t) v(t) d x\right|_{t_{1}} ^{t_{2}}+2 \int_{t_{1}}^{t_{2}}\left\|u_{t}(t)\right\|_{2}^{2} d t+2 \int_{t_{1}}^{t_{2}}\left\|v_{t}(t)\right\|_{2}^{2} d t \\
& +\frac{1}{2} \int_{t_{1}}^{t_{2}}\left(g_{1} \circ \nabla u\right)(t) d t+\frac{1}{2} \int_{t_{1}}^{t_{2}}\left(g_{2} \circ \nabla v\right)(t) d t-\int_{t_{1}}^{t_{2}}\left(\nabla u(t), \nabla u_{t}(t)\right) d t \\
& -\int_{t_{1}}^{t_{2}}\left(\nabla v(t), \nabla v_{t}(t)\right) d t+\frac{1}{2} \int_{t_{1}}^{t_{2}} \int_{0}^{t} g_{1}(t-\tau)\|\nabla u(\tau)\|_{2}^{2} d \tau d t \\
& +\frac{1}{2} \int_{t_{1}}^{t_{2}} \int_{0}^{t} g_{2}(t-\tau)\|\nabla v(\tau)\|_{2}^{2} d \tau d t .
\end{aligned}
$$

Now we estimate every term of the right-hand side of the (3.13). First, by Hölder's inequality and Poincaré's inequality

$$
\begin{aligned}
\int_{\Omega}\left|u(t) u_{t}(t)\right| d x+\int_{\Omega}\left|v(t) v_{t}(t)\right| d x & \leq \frac{1}{2}\|u(t)\|_{2}^{2}+\frac{1}{2}\left\|u_{t}(t)\right\|_{2}^{2}+\frac{1}{2}\|v(t)\|_{2}^{2}+\frac{1}{2}\left\|v_{t}(t)\right\|_{2}^{2} \\
& \leq \frac{\lambda}{2}\|\nabla u(t)\|_{2}^{2}+\frac{1}{2}\left\|u_{t}(t)\right\|_{2}^{2}+\frac{\lambda}{2}\|\nabla v(t)\|_{2}^{2}+\frac{1}{2}\left\|v_{t}(t)\right\|_{2}^{2}
\end{aligned}
$$

where $\lambda$ being the first eigenvalue of the operator $-\Delta$ under homogeneous Dirichlet boundary conditions. Then, by (3.9), we see that

$$
\int_{\Omega}\left|u(t) u_{t}(t)\right| d x+\int_{\Omega}\left|v(t) v_{t}(t)\right| d x \leq c_{1} E(t),
$$

where $c_{1}$ is a constant independent on $u$ and $v$, from which follows that

$$
\left.\int_{\Omega}\left|u(t) u_{t}(t)\right| d x\right|_{t_{1}} ^{t_{2}}+\left.\int_{\Omega}\left|v(t) v_{t}(t)\right| d x\right|_{t_{1}} ^{t_{2}} \leq 2 c_{1} E\left(t_{1}\right) .
$$

Since $0 \leq J(t) \leq E(t)$, from (3.2) we deduce that

$$
\int_{t_{1}}^{t_{2}}\left(\left\|\nabla u_{t}(t)\right\|_{2}^{2}+\left\|\nabla v_{t}(t)\right\|_{2}^{2}\right) d t \leq E\left(t_{1}\right) .
$$

Hence, by Poincaré inequality we get

$$
2 \int_{t_{1}}^{t_{2}}\left\|u_{t}(t)\right\|_{2}^{2} d t+2 \int_{t_{1}}^{t_{2}}\left\|v_{t}(t)\right\|_{2}^{2} d t \leq 2 c_{2} E\left(t_{1}\right),
$$

where $c_{2}$ is a constant independent on $u$ and $v$. In addition, using Young's inequality for convolution $\left\|f^{*} g\right\|_{q} \leq\|f\|_{r}\|g\|_{s}$ with $1 / q=1 / r+1 / s-1$ and $1 \leq q, r, s \leq \infty$, noting that if $q=1$, then $r=1$ and $s=1$, we have 


$$
\begin{aligned}
\int_{t_{1}}^{t_{2}} \int_{0}^{t} g_{1}(t-\tau)\|\nabla u(\tau)\|_{2}^{2} d \tau d t & =\left\|g_{1} *\right\| \nabla u\left\|_{2}^{2}\right\|_{1} \leq\left\|g_{1}\right\|_{1}\|\| \nabla u\left\|_{2}^{2}\right\|_{1} \\
& =\int_{t_{1}}^{t_{2}} g_{1}(t) d t \int_{t_{1}}^{t_{2}}\|\nabla u(t)\|_{2}^{2} d t \\
& \leq\left(1-k_{1}\right) \int_{t_{1}}^{t_{2}}\|\nabla u(t)\|_{2}^{2} d t
\end{aligned}
$$

and

$$
\begin{aligned}
\int_{t_{1}}^{t_{2}} \int_{0}^{t} g_{2}(t-\tau)\|\nabla v(\tau)\|_{2}^{2} d \tau d t & =\left\|g_{2} *\right\| \nabla v\left\|_{2}^{2}\right\|_{1} \leq\left\|g_{2}\right\|_{1}\|\| \nabla v\left\|_{2}^{2}\right\|_{1} \\
& =\int_{t_{1}}^{t_{2}} g_{2}(t) d t \int_{t_{1}}^{t_{2}}\|\nabla v(t)\|_{2}^{2} d t \\
& \leq\left(1-k_{2}\right) \int_{t_{1}}^{t_{2}}\|\nabla v(t)\|_{2}^{2} d t .
\end{aligned}
$$

Hence, combining (3.9), (3.16) with (3.17) we then have

$$
\begin{aligned}
& \int_{t_{1}}^{t_{2}} \int_{0}^{t} g_{1}(t-\tau)\|\nabla u(\tau)\|_{2}^{2} d \tau d t+\int_{t_{1}}^{t_{2}} \int_{0}^{t} g_{2}(t-\tau)\|\nabla v(\tau)\|_{2}^{2} d \tau d t \\
& \leq\left(1-k_{1}\right) \int_{t_{1}}^{t_{2}}\|\nabla u(t)\|_{2}^{2} d t+\left(1-k_{2}\right) \int_{t_{1}}^{t_{2}}\|\nabla v(t)\|_{2}^{2} d t \\
& \leq(1-k) \int_{t_{1}}^{t_{2}}\left(\|\nabla u(t)\|_{2}^{2}+\|\nabla v(t)\|_{2}^{2}\right) d t \leq \frac{2(1-k)(p+2)}{k(p+1)} \int_{t_{1}}^{t_{2}} E(t) d t .
\end{aligned}
$$

From (3.9), we also have

$$
\begin{aligned}
& \int_{t_{1}}^{t_{2}} \int_{0}^{t} g_{1}(t-\tau)\|\nabla u(t)\|_{2}^{2} d \tau d t+\int_{t_{1}}^{t_{2}} \int_{0}^{t} g_{2}(t-\tau)\|\nabla v(t)\|_{2}^{2} d \tau d t \\
& \leq\left(1-k_{1}\right) \int_{t_{1}}^{t_{2}}\|\nabla u(t)\|_{2}^{2} d t+\left(1-k_{2}\right) \int_{t_{1}}^{t_{2}}\|\nabla v(t)\|_{2}^{2} d t \\
& \leq(1-k) \int_{t_{1}}^{t_{2}}\left(\|\nabla u(t)\|_{2}^{2}+\|\nabla v(t)\|_{2}^{2}\right) d t \leq \frac{2(1-k)(p+2)}{k(p+1)} \int_{t_{1}}^{t_{2}} E(t) d t .
\end{aligned}
$$

Combining (3.18) with (3.19), we deduce that

$$
\begin{aligned}
& \frac{1}{2} \int_{t_{1}}^{t_{2}}\left(g_{1} \circ \nabla u\right)(t) d t+\frac{1}{2} \int_{t_{1}}^{t_{2}}\left(g_{2} \circ \nabla v\right)(t) d t \\
& \leq \int_{t_{1}}^{t_{2}} \int_{0}^{t} g_{1}(t-\tau)\left(\|\nabla u(\tau)\|_{2}^{2}+\|\nabla u(t)\|_{2}^{2}\right) d \tau d t+\int_{t_{1}}^{t_{2}} \int_{0}^{t} g_{2}(t-\tau) \\
& \left(\|\nabla v(\tau)\|_{2}^{2}+\|\nabla v(t)\|_{2}^{2}\right) d \tau d t \leq \frac{4(1-k)(p+2)}{k(p+1)} \int_{t_{1}}^{t_{2}} E(t) d t .
\end{aligned}
$$


Finally, we also have the following estimate

$$
\begin{aligned}
& \int_{t_{1}}^{t_{2}}\left(\nabla u(t), \nabla u_{t}(t)\right) d t+\int_{t_{1}}^{t_{2}}\left(\nabla v(t), \nabla v_{t}(t)\right) d t \\
& =\frac{1}{2} \int_{t_{1}}^{t_{2}} \frac{d}{d t}\|\nabla u(t)\|_{2}^{2} d t+\frac{1}{2} \int_{t_{1}}^{t_{2}} \frac{d}{d t}\|\nabla v(t)\|_{2}^{2} d t \\
& =\frac{1}{2}\left(\left\|\nabla u\left(t_{2}\right)\right\|_{2}^{2}-\left\|\nabla u\left(t_{1}\right)\right\|_{2}^{2}\right)+\frac{1}{2}\left(\left\|\nabla v\left(t_{2}\right)\right\|_{2}^{2}-\left\|\nabla v\left(t_{1}\right)\right\|_{2}^{2}\right) \\
& \leq \frac{2(p+2)}{k(p+1)} E\left(t_{1}\right) \leq c_{3} E\left(t_{1}\right) .
\end{aligned}
$$

where $c_{3}$ is a constant independent on $u$ and $v$. Combining (3.13)-(3.21), we obtain

$$
2 \int_{t_{1}}^{t_{2}} E(t) d t-2(p+1) \int_{t_{1}}^{t_{2}} \int_{\Omega} F(u, v) d x d t \leq C E\left(t_{1}\right)+\frac{5(1-k)(p+2)}{k(p+1)} \int_{t_{1}}^{t_{2}} E(t) d t .
$$

where $C$ is a constant independent on $u$.

On the other hand, from (3.3) and (3.9), we have

$$
\begin{aligned}
2(p+1) \int_{\Omega} F(u, v) d x & =\frac{p+1}{p+2}\left(\|u+v\|_{2(p+2)}^{2(p+2)}+2\|u v\|_{(p+2)}^{(p+2)}\right) \\
& \leq \frac{p+1}{p+2} \eta\left(k_{1}\|\nabla u\|_{2}^{2}+k_{2}\|\nabla v\|_{2}^{2}\right)^{(p+2)} \\
& \leq 2 \eta\left(\frac{2(p+2)}{(p+1)} E(0)\right)^{(p+1)} E(t)
\end{aligned}
$$

which implies

$$
2 \int_{t_{1}}^{t_{2}} E(t) d t-2(p+1) \int_{t_{1}}^{t_{2}} \int_{\Omega} F(u, v) d x d t \geq 2\left(1-\eta\left(\frac{2(p+2)}{(p+1)} E(0)\right)^{(p+1)}\right) \int_{t_{1}}^{t_{2}} E(t) d t .
$$

Note that $E(0)<E_{1}$, we see that

$$
1-\eta\left(\frac{2(p+2)}{(p+1)} E(0)\right)^{(p+1)}>0 .
$$

Thus, combining (3.22) with (3.23), we have

$$
2\left(1-\eta\left(\frac{2(p+2)}{(p+1)} E(0)\right)^{(p+1)}\right) \int_{t_{1}}^{t_{2}} E(t) d t \leq C E\left(t_{1}\right)+\frac{5(1-k)(p+2)}{k(p+1)} \int_{t_{1}}^{t_{2}} E(t) d t
$$

that is

$$
2\left(1-\eta\left(\frac{2(p+2)}{(p+1)} E(0)\right)^{(p+1)}-\frac{5(1-k)(p+2)}{2 k(p+1)}\right) \int_{t_{1}}^{t_{2}} E(t) d t \leq C E\left(t_{1}\right) .
$$

Denote

$$
a=2\left(1-\eta\left(\frac{2(p+2)}{(p+1)} E(0)\right)^{(p+1)}-\frac{5(1-k)(p+2)}{2 k(p+1)}\right) .
$$


We rewrite (3.24)

$$
a \int_{t}^{\infty} E(\tau) d \tau \leq C E(t)
$$

for every $t \in[0, \infty)$.

Since $a>0$ from the assumption conditions, by Lemma 3.4, we obtain the following energy decay for problem (1.1) as

$$
E(t)<E(0) \exp \left(1-a C^{-1} t\right)
$$

for every $t \geq \mathrm{Ca}^{-1}$. $\square$

\section{Blow-up of solution}

In this section, we deal with the blow-up solutions of the system (1.1). Set

$$
\theta_{i}=k_{i}-\frac{1}{4(p+2)(p+1)} \int_{0}^{\infty} g_{i}(s) d s, \quad i=1,2 .
$$

From the assumption (G2), we have $\theta_{i}>0(i=1,2)$. Similarly Lemma 3.2, we have

Lemma 4.1. Assume (2.1) holds. Then there exists $\eta_{1}>0$ such that for any $(u, v) \in H_{0}^{1}(\Omega) \times H_{0}^{1}(\Omega)$, we have

$$
\|u+v\|_{2(p+2)}^{2(p+2)}+2\|u v\|_{p+2}^{p+2} \leq \eta_{1}\left(\theta_{1}\|\nabla u(t)\|_{2}^{2}+\theta_{2}\|\nabla v(t)\|_{2}^{2}\right)^{p+2},
$$

where the constants $\theta_{i}(i=1,2)$ are defined in (4.1).

To prove our result and for the sake of simplicity, we take $a=b=1$ and introduce the following:

$$
B_{1}=\eta_{1}^{\frac{1}{2(p+2)}}, \quad \alpha_{*}=B_{1}^{-\frac{p+2}{p+1}}, \quad E_{2}=\left(\frac{1}{2}-\frac{1}{2(p+2)}\right) \alpha_{*}^{2} .
$$

Then we have

Lemma 4.2. Let (G1), G(2) and (2.1) hold. Let $(u, v)$ be the solution of the system (1.1). Assume further that $E(0)<E_{2}$ and

$$
\left(\theta_{1}\left\|\nabla u_{0}\right\|_{2}^{2}+\theta_{2}\left\|\nabla v_{0}\right\|_{2}^{2}\right)^{1 / 2}>\alpha_{* \prime}
$$

where the constants $\theta_{i}(i=1,2)$ are defined in (4.1). Then there exists a constant $\alpha_{2}$ $>\alpha$ such that

$$
\left(\theta_{1}\|\nabla u(t)\|_{2}^{2}+\theta_{2}\|\nabla v(t)\|_{2}^{2}\right)^{1 / 2} \geq \alpha_{2}, \quad \text { for } t \in(0, T) .
$$

Proof. We first note that, by (2.5), (4.2) and the definition of $B_{1}$, we have

$$
\begin{aligned}
E(t) & \geq \frac{1}{2}\left(\theta_{1}\|\nabla u(t)\|_{2}^{2}+\theta_{2}\|\nabla v(t)\|_{2}^{2}\right)-\frac{1}{2(p+2)}\left(\|u+v\|_{2(p+2)}^{2(p+2)}+2\|u v\|_{p+2}^{p+2}\right) \\
& \geq \frac{1}{2}\left(\theta_{1}\|\nabla u(t)\|_{2}^{2}+\theta_{2}\|\nabla v(t)\|_{2}^{2}\right)-\frac{B_{1}^{2(p+2)}}{2(p+2)}\left(\theta_{1}\|\nabla u(t)\|_{2}^{2}+\theta_{2}\|\nabla v(t)\|_{2}^{2}\right)^{p+2} \\
& =\frac{1}{2} \alpha^{2}-\frac{B_{1}^{2(p+2)}}{2(p+2)} \alpha^{2(p+2)},
\end{aligned}
$$


where $\alpha=\left(\theta_{1}\|\nabla u(t)\|_{2}^{2}+\theta_{2}\|\nabla v(t)\|_{2}^{2}\right)^{1 / 2}$. It is not hard to verify that $g$ is increasing for $0<\alpha<\alpha_{*}$, decreasing for $\alpha>\alpha *, g(\alpha) \rightarrow-\infty$ as $\alpha \rightarrow+\infty$, and

$$
g\left(\alpha_{*}\right)=\frac{1}{2} \alpha_{*}^{2}-\frac{B_{1}^{2(p+2)}}{2(p+2)} \alpha_{*}^{2(p+2)}=E_{2}
$$

where $\alpha *$ is given in (4.3). Since $E(0)<E_{2}$, there exists $\alpha_{2}>\alpha *$ such that $g\left(\alpha_{2}\right)=E(0)$. Set $\alpha_{0}=\left(\theta_{1}\left\|\nabla u_{0}\right\|_{2}^{2}+\theta_{2}\left\|\nabla v_{0}\right\|_{2}^{2}\right)^{1 / 2}$, then by (4.6) we get $g\left(\alpha_{0}\right) \leq E(0)=g\left(\alpha_{2}\right)$, which implies that $\alpha_{0} \geq \alpha_{2}$. Now, to establish (4.5), we suppose by contradiction that

$$
\left(\theta_{1}\left\|\nabla u\left(t_{0}\right)\right\|_{2}^{2}+\theta_{2}\left\|\nabla v\left(t_{0}\right)\right\|_{2}^{2}\right)^{1 / 2}<\alpha_{2}
$$

for some $t_{0}>0$. By the continuity of $\theta_{1}\|\nabla u(t)\|_{2}^{2}+\theta_{2}\|\nabla v(t)\|_{2}^{2}$ we can choose $t_{0}$ such that

$$
\left(\theta_{1}\left\|\nabla u\left(t_{0}\right)\right\|_{2}^{2}+\theta_{2}\left\|\nabla v\left(t_{0}\right)\right\|_{2}^{2}\right)^{1 / 2}>\alpha_{*} .
$$

Again, the use of (4.6) leads to

$$
E\left(t_{0}\right) \geq g\left(\left(\theta_{1}\left\|\nabla u\left(t_{0}\right)\right\|_{2}^{2}+\theta_{2}\left\|\nabla v\left(t_{0}\right)\right\|_{2}^{2}\right)^{1 / 2}\right)>g\left(\alpha_{2}\right)=E(0) .
$$

This is impossible since $E(t) \leq E(0)$ for all $t \in[0, T)$. Hence (4.5) is established.

Theorem 4.3. Assume (G1), (G2) and (2.1) hold. Then any solution of problem (1.1) with initial data satisfying

$$
\left(\theta_{1}\left\|\nabla u_{0}\right\|_{2}^{2}+\theta_{2}\left\|\nabla v_{0}\right\|_{2}^{2}\right)^{1 / 2}>\alpha_{*} \text { and } E(0)<E_{2}
$$

blows up in finite time, where the constants $\theta_{i}(i=1,2)$ are defined in (4.1) and $\alpha_{*}$, $E_{2}$ are defined in (4.3).

Proof. Assume by contradiction that the solution $(u, v)$ is global. Then, for any $T>0$ we consider $H(t):[0, T] \rightarrow \mathbb{R}_{+}$defined by

$$
H(t)=\|u(t)\|_{2}^{2}+\|v(t)\|_{2}^{2}+\int_{0}^{t}\|\nabla u(\tau)\|_{2}^{2} d \tau+\int_{0}^{t}\|\nabla v(\tau)\|_{2}^{2} d \tau+(T-t)\left(\left\|\nabla u_{0}\right\|_{2}^{2}+\left\|\nabla v_{0}\right\|_{2}^{2}\right)+\beta\left(t+s_{0}\right)^{2},
$$

where $\beta$ and $s_{0}$ are positive constants to be determined later. A direct computation yields

$$
\begin{aligned}
H^{\prime}(t)= & 2 \int_{\Omega} u(t) u_{t}(t) d x+2 \int_{\Omega} v(t) v_{t}(t) d x+\|\nabla u(t)\|_{2}^{2}+\|\nabla v(t)\|_{2}^{2} \\
& -\left\|\nabla u_{0}\right\|_{2}^{2}-\left\|\nabla v_{0}\right\|_{2}^{2}+2 \beta\left(t+s_{0}\right) \\
= & 2 \int_{\Omega} u(t) u_{t}(t) d x+2 \int_{\Omega} v(t) v_{t}(t) d x+2 \int_{0}^{t}\left(\nabla u(\tau), \nabla u_{t}(\tau)\right) d \tau \\
& +2 \int_{0}^{t}\left(\nabla v(\tau), \nabla v_{t}(\tau)\right) d \tau+2 \beta\left(t+s_{0}\right)
\end{aligned}
$$

and

$$
\begin{aligned}
H^{\prime \prime}(t)= & 2 \int_{\Omega} u(t) u_{t t}(t) d x+2 \int_{\Omega} v(t) v_{t t}(t) d x+2\left\|u_{t}(t)\right\|_{2}^{2}+2\left\|v_{t}(t)\right\|_{2}^{2} \\
& +2\left(\nabla u(t), \nabla u_{t}(t)\right)+2\left(\nabla v(t), \nabla v_{t}(t)\right)+2 \beta \text { for a.e. } t \in[0, T]
\end{aligned}
$$

Multiplying the first equation of system (1.1) by $u$ and the second equation of system (1.1) by $v$, integrating over $\Omega$, using integration by parts and summing up, we have 


$$
\begin{aligned}
& \left(u_{t t}, u(t)\right)+\left(v_{t t}, v(t)\right)+\left(\nabla u(t), \nabla u_{t}(t)\right)+\left(\nabla v(t), \nabla v_{t}(t)\right) \\
= & -\|\nabla u(t)\|_{2}^{2}-\|\nabla v(t)\|_{2}^{2}-\int_{\Omega} \int_{0}^{t} g_{1}(t-\tau) \Delta u(\tau) d \tau u(t) d x \\
& -\int_{\Omega} \int_{0}^{t} g_{2}(t-\tau) \Delta v(\tau) d \tau v(t) d x+2(p+2) \int_{\Omega} F(u, v) d x,
\end{aligned}
$$

which implies

$$
\begin{aligned}
H^{\prime \prime}(t)= & 2\left\|u_{t}(t)\right\|_{2}^{2}+2\left\|v_{t}(t)\right\|_{2}^{2}-2\|\nabla u(t)\|_{2}^{2}-2\|\nabla v(t)\|_{2}^{2}+4(p+2) \int_{\Omega} F(u, v) d x \\
& -2 \int_{\Omega} \int_{0}^{t} g_{1}(t-\tau) \Delta u(\tau) d \tau u(t) d x-2 \int_{\Omega} \int_{0}^{t} g_{2}(t-\tau) \Delta v(\tau) d \tau v(t) d x+2 \beta .
\end{aligned}
$$

Therefore, we have

$$
\begin{aligned}
& H(t) H^{\prime \prime}(t)-\frac{p+3}{2} H^{\prime}(t)^{2} \\
= & 2 H(t)\left(\left\|u_{t}(t)\right\|_{2}^{2}+\left\|v_{t}(t)\right\|_{2}^{2}-\|\nabla u(t)\|_{2}^{2}-\|\nabla v(t)\|_{2}^{2}+2(p+2) \int_{\Omega} F(u, v) d x+2 \beta\right) \\
& -2 H(t)\left(\int_{\Omega} \int_{0}^{t} g_{1}(t-\tau) \Delta u(\tau) d \tau u(t) d x+\int_{\Omega} \int_{0}^{t} g_{2}(t-\tau) \Delta v(\tau) d \tau v(t) d x\right) \\
& -2(p+3)\left(\int_{\Omega} u(t) u_{t}(t) d x+\int_{\Omega} v(t) v_{t}(t) d x+\int_{0}^{t}\left(\nabla u(\tau), \nabla u_{t}(\tau)\right) d \tau\right. \\
& \left.+\int_{0}^{t}\left(\nabla v(\tau), \nabla v_{t}(\tau)\right) d \tau+\beta\left(t+s_{0}\right)\right)^{2} \\
= & 2 H(t)\left(\left\|u_{t}(t)\right\|_{2}^{2}+\left\|v_{t}(t)\right\|_{2}^{2}-\|\nabla u(t)\|_{2}^{2}-\|\nabla v(t)\|_{2}^{2}+2(p+2) \int_{\Omega} F(u, v) d x+2 \beta\right) \\
& -2 H(t)\left(\int_{\Omega} \int_{0}^{t} g_{1}(t-\tau) \Delta u(\tau) d \tau u(t) d x+\int_{\Omega} \int_{0}^{t} g_{2}(t-\tau) \Delta v(\tau) d \tau v(t) d x\right) \\
& +2(p+3)\left(G(t)-\left(H(t)-(T-t)\left(\left\|\nabla u_{0}\right\|_{2}^{2}+\left\|\nabla v_{0}\right\|_{2}^{2}\right)\right) \Psi(t)\right),
\end{aligned}
$$

where $\Psi(t), G(t):[0, T] \rightarrow \mathbb{R}_{+}$are the functions defined by

$$
\Psi(t)=\left\|u_{t}(t)\right\|_{2}^{2}+\left\|v_{t}(t)\right\|_{2}^{2}+\int_{0}^{t}\left\|\nabla u_{t}(\tau)\right\|_{2}^{2} d \tau+\int_{0}^{t}\left\|\nabla v_{t}(\tau)\right\|_{2}^{2} d \tau+\beta
$$

and

$$
\begin{aligned}
G(t)= & \left(\|u(t)\|_{2}^{2}+\|v(t)\|_{2}^{2}+\int_{0}^{t}\|\nabla u(\tau)\|_{2}^{2} d \tau+\int_{0}^{t}\|\nabla v(\tau)\|_{2}^{2} d \tau+\beta\left(t+s_{0}\right)^{2}\right) \Psi(t) \\
& -\left(\int_{\Omega} u(t) u_{t}(t) d x+\int_{\Omega} v(t) v_{t}(t) d x+\int_{0}^{t}\left(\nabla u(\tau), \nabla u_{t}(\tau)\right) d \tau\right. \\
& \left.+\int_{0}^{t}\left(\nabla v(\tau), \nabla v_{t}(\tau)\right) d \tau+\beta\left(t+s_{0}\right)\right)^{2} .
\end{aligned}
$$


Using the Schwarz inequality, we have

$$
\begin{aligned}
&\left(\int_{\Omega} u(t) u_{t}(t) d x\right)^{2} \leq\|u(t)\|_{2}^{2}\left\|u_{t}(t)\right\|_{2^{\prime}}^{2} \quad\left(\int_{\Omega} v(t) v_{t}(t) d x\right)^{2} \leq\|v(t)\|_{2}^{2}\left\|v_{t}(t)\right\|_{2^{\prime}}^{2} \\
&\left(\int_{0}^{t}\left(\nabla u(\tau), \nabla u_{t}(\tau)\right) d \tau\right)^{2} \leq \int_{0}^{t}\|\nabla u(\tau)\|_{2}^{2} d \tau \int_{0}^{t}\left\|\nabla u_{t}(\tau)\right\|_{2}^{2} d \tau \\
&\left(\int_{0}^{t}\left(\nabla v(\tau), \nabla v_{t}(\tau)\right) d \tau\right)^{2} \leq \int_{0}^{t}\|\nabla v(\tau)\|_{2}^{2} d \tau \int_{0}^{t}\left\|\nabla v_{t}(\tau)\right\|_{2}^{2} d \tau \\
& \int_{\Omega} u(t) u_{t}(t) d x \int_{\Omega} v(t) v_{t}(t) d x \leq\|u(t)\|_{2}\left\|v_{t}(t)\right\|_{2}\left\|u_{t}(t)\right\|\left\|_{2}\right\| v(t) \|_{2} \\
& \leq \frac{1}{2}\|u(t)\|_{2}^{2}\left\|v_{t}(t)\right\|_{2}^{2}+\frac{1}{2}\left\|u_{t}(t)\right\|_{2}^{2}\|v(t)\|_{2}^{2}, \\
& \beta\left(t+s_{0}\right) \int_{\Omega} u(t) u_{t}(t) d x \leq \sqrt{\beta} \sqrt{\beta}\left(t+s_{0}\right)\|u(t)\|_{2}\left\|u_{t}(t)\right\|_{2} \\
& \leq \frac{1}{2} \beta\|u(t)\|_{2}^{2}+\frac{1}{2} \beta\left(t+s_{0}\right)^{2}\left\|u_{t}(t)\right\|_{2}^{2},
\end{aligned}
$$

and

$$
\beta\left(t+s_{0}\right) \int_{\Omega} v(t) v_{t}(t) d x \leq \frac{1}{2} \beta\|v(t)\|_{2}^{2}+\frac{1}{2} \beta\left(t+s_{0}\right)^{2}\left\|v_{t}(t)\right\|_{2}^{2} .
$$

Similarly, we have

$$
\begin{aligned}
& \int_{\Omega} u(t) u_{t}(t) d x \int_{0}^{t}\left(\nabla u(\tau), \nabla u_{t}(\tau)\right) d \tau \\
& \int_{\Omega} u(t) u_{t}(t) d x \int_{0}^{t}\left(\nabla v(\tau), \nabla v_{t}(\tau)\right) d \tau \leq \frac{1}{2}\|u(t)\|_{2}^{2} \int_{0}^{2} \int_{0}^{t}\left\|\nabla u_{t}(\tau)\right\|_{2}^{2} d \tau+\frac{1}{2}\left\|v_{t}(\tau)\right\|_{2}^{2} d \tau+\frac{1}{2}\|\|_{t}(t)\left\|_{2}^{2} \int_{0}^{t}\right\| \nabla u(\tau) \|_{2}^{2} d \tau, \\
& \int_{\Omega} v(t) v_{t}(t) d x \int_{0}^{t}\left(\nabla u(\tau), \nabla u_{t}(\tau)\right) d \tau \leq \frac{1}{2}\|v(t)\|_{2}^{2} \int_{0}^{t}\left\|\nabla u_{t}(\tau)\right\|_{2}^{2} d \tau+\frac{1}{2}\left\|v_{t}(t)\right\|_{2}^{2} \int_{0}^{t}\|\nabla u(\tau)\|_{2}^{2} d \tau, \\
& \int_{\Omega} v(t) v_{t}(t) d x \int_{0}^{t}\left(\nabla v(\tau), \nabla v_{t}(\tau)\right) d \tau \leq \frac{1}{2}\|v(t)\|_{2}^{2} \int_{0}^{t}\left\|\nabla v_{t}(\tau)\right\|_{2}^{2} d \tau+\frac{1}{2}\left\|v_{t}(t)\right\|_{2}^{2} \int_{0}^{t}\|\nabla v(\tau)\|_{2}^{2} d \tau,
\end{aligned}
$$

and

$$
\begin{gathered}
\int_{0}^{t}\left(\nabla u(\tau), \nabla u_{t}(\tau)\right) d \tau \int_{0}^{t}\left(\nabla v(\tau), \nabla v_{t}(\tau)\right) d \tau \\
\leq \frac{1}{2} \int_{0}^{t}\|\nabla u(\tau)\|_{2}^{2} d \tau \int_{0}^{t}\left\|\nabla v_{t}(\tau)\right\|_{2}^{2} d \tau+\frac{1}{2} \int_{0}^{t}\left\|\nabla u_{t}(\tau)\right\|_{2}^{2} d \tau \int_{0}^{t}\|\nabla v(\tau)\|_{2}^{2} d \tau, \\
\beta\left(t+s_{0}\right) \int_{0}^{t}\left(\nabla u(\tau), \nabla u_{t}(\tau)\right) d \tau \leq \frac{1}{2} \beta \int_{0}^{t}\|\nabla u(\tau)\|_{2}^{2} d \tau+\frac{1}{2} \beta\left(t+s_{0}\right)^{2} \int_{0}^{t}\left\|\nabla u_{t}(\tau)\right\|_{2}^{2} d \tau, \\
\beta\left(t+s_{0}\right) \int_{0}^{t}\left(\nabla v(\tau), \nabla v_{t}(\tau)\right) d \tau \leq \frac{1}{2} \beta \int_{0}^{t}\|\nabla v(\tau)\|_{2}^{2} d \tau+\frac{1}{2} \beta\left(t+s_{0}\right)^{2} \int_{0}^{t}\left\|\nabla v_{t}(\tau)\right\|_{2}^{2} d \tau .
\end{gathered}
$$

The previous inequalities entail $G(t) \geq 0$ for every $[0, T]$. Using (4.7), we get

$$
H(t) H^{\prime \prime}(t)-\frac{p+3}{2} H^{\prime}(t)^{2} \geq H(t) L(t) \text { for a.e. } t \in[0, T]
$$

where

$$
\begin{aligned}
L(t)= & -2(p+2)\left(\left\|u_{t}(t)\right\|_{2}^{2}+\left\|v_{t}(t)\right\|_{2}^{2}\right)-2\|\nabla u(t)\|_{2}^{2}-2\|\nabla v(t)\|_{2}^{2}+4(p+2) \int_{\Omega} F(u, v) d x \\
& -2\left(\int_{\Omega} \int_{0}^{t} g_{1}(t-\tau) \Delta u(\tau) d \tau u(t) d x+\int_{\Omega} \int_{0}^{t} g_{2}(t-\tau) \Delta v(\tau) d \tau v(t) d x\right) \\
& -2(p+3)\left(\int_{0}^{t}\left\|\nabla u_{t}(\tau)\right\|_{2}^{2} d \tau+\int_{0}^{t}\left\|\nabla v_{t}(\tau)\right\|_{2}^{2} d \tau\right)-2(p+1) \beta .
\end{aligned}
$$


For the fifth term on the right-hand side of (4.9), we have

$$
\begin{aligned}
& -\int_{\Omega} \int_{0}^{t} g_{1}(t-\tau) \Delta u(\tau) d \tau u(t) d x=\int_{0}^{t} g_{1}(t-\tau) \int_{\Omega} \nabla u(\tau) \nabla u(t) d x d \tau \\
= & \int_{0}^{t} g_{1}(t-\tau) \int_{\Omega} \nabla u(t) \nabla(u(\tau)-u(t)) d x d \tau+\int_{0}^{t} g_{1}(t-\tau)\|\nabla u(t)\|_{2}^{2} d \tau \\
= & \int_{0}^{t} g_{1}(t-\tau) \int_{\Omega} \nabla u(t) \nabla(u(\tau)-u(t)) d x d \tau+\int_{0}^{t} g_{1}(\tau)\|\nabla u(t)\|_{2}^{2} d \tau .
\end{aligned}
$$

Similarly,

$$
\begin{aligned}
& -\int_{\Omega} \int_{0}^{t} g_{2}(t-\tau) \Delta v(\tau) d \tau v(t) d x \\
= & \int_{0}^{t} g_{2}(t-\tau) \int_{\Omega} \nabla v(t) \nabla(v(\tau)-v(t)) d x d \tau+\int_{0}^{t} g_{2}(\tau)\|\nabla v(t)\|_{2}^{2} d \tau .
\end{aligned}
$$

Combining (4.9), (4.10) with (4.11), we get

$$
\begin{aligned}
L(t)= & -2(p+2)\left(\left\|u_{t}(t)\right\|_{2}^{2}+\left\|v_{t}(t)\right\|_{2}^{2}\right)-2\left(1-\int_{0}^{t} g_{1}(\tau) d \tau\right)\|\nabla u(t)\|_{2}^{2} \\
& -2\left(1-\int_{0}^{t} g_{2}(\tau) d \tau\right)\|\nabla v(t)\|_{2}^{2}+4(p+2) \int_{\Omega} F(u, v) d x-2(p+1) \beta \\
& +2 \int_{0}^{t} g_{1}(t-\tau) \int_{\Omega} \nabla u(t) \nabla(u(\tau)-u(t)) d x d \tau-2(p+3) \int_{0}^{t}\left\|\nabla u_{t}(\tau)\right\|_{2}^{2} d \tau \\
& +2 \int_{0}^{t} g_{2}(t-\tau) \int_{\Omega} \nabla v(t) \nabla(v(\tau)-v(t)) d x d \tau-2(p+3) \int_{0}^{t}\left\|\nabla v_{t}(\tau)\right\|_{2}^{2} d \tau .
\end{aligned}
$$

Since

$$
\begin{aligned}
& 2 \int_{0}^{t} g_{1}(t-\tau) \int_{\Omega} \nabla u(t) \nabla(u(\tau)-u(t)) d x d \tau \\
\geq & -2\left((p+2) \int_{0}^{t} g_{1}(t-\tau)\|\nabla u(\tau)-\nabla u(t)\|_{2}^{2} d \tau+\frac{1}{4(p+2)} \int_{0}^{t} g_{1}(\tau)\|\nabla u(t)\|_{2}^{2} d \tau\right) \\
= & -2(p+2)\left(g_{1} \circ \nabla u\right)(t)-\frac{1}{2(p+2)} \int_{0}^{t} g_{1}(\tau)\|\nabla u(t)\|_{2}^{2} d \tau,
\end{aligned}
$$

and

$$
\begin{aligned}
& 2 \int_{0}^{t} g_{2}(t-\tau) \int_{\Omega} \nabla v(t) \nabla(v(\tau)-v(t)) d x d \tau \\
\geq & -2(p+2)\left(g_{2} \circ \nabla v\right)(t)-\frac{1}{2(p+2)} \int_{0}^{t} g_{2}(\tau)\|\nabla v(t)\|_{2}^{2} d \tau,
\end{aligned}
$$


inserting (4.13) and (4.14) into (4.12), we have

$$
\begin{aligned}
L(t) \geq & -2(p+2)\left(\left\|u_{t}(t)\right\|_{2}^{2}+\left\|v_{t}(t)\right\|_{2}^{2}+\left(g_{1} \circ \nabla u\right)(t)+\left(g_{2} \circ \nabla v\right)(t)\right)+4(p+2) \int_{\Omega} F(u, v) d x \\
& -2\left(1-\int_{0}^{t} g_{1}(\tau) d \tau+\frac{1}{4(p+2)} \int_{0}^{t} g_{1}(\tau) d \tau\right)\|\nabla u(t)\|_{2}^{2}-2\left(1-\int_{0}^{t} g_{2}(\tau) d \tau\right)\|\nabla v(t)\|_{2}^{2} \\
& -\frac{1}{2(p+2)} \int_{0}^{t} g_{2}(\tau)\|\nabla v(t)\|_{2}^{2} d \tau-2(p+3)\left(\int_{0}^{t}\left\|\nabla v_{t}(\tau)\right\|_{2}^{2} d \tau\right. \\
& \left.+\int_{0}^{t}\left\|\nabla u_{t}(\tau)\right\|_{2}^{2} d \tau\right)-2(p+1) \beta \\
\geq & -4(p+2) E(t)+2(p+1)\left(1-\int_{0}^{t} g_{1}(\tau) d \tau\right)\|\nabla u(t)\|_{2}^{2}-\frac{1}{2(p+2)} \int_{0}^{t} g_{1}(\tau)\|\nabla u(t)\|_{2}^{2} d \tau \\
& +2(p+1)\left(1-\int_{0}^{t} g_{2}(\tau) d \tau\right)\|\nabla v(t)\|_{2}^{2}-\frac{1}{2(p+2)} \int_{0}^{t} g_{2}(\tau)\|\nabla v(t)\|_{2}^{2} d \tau \\
& -2(p+3)\left(\int_{0}^{t}\left\|\nabla v_{t}(\tau)\right\|_{2}^{2} d \tau+\int_{0}^{t}\left\|\nabla u_{t}(\tau)\right\|_{2}^{2} d \tau\right)-2(p+1) \beta .
\end{aligned}
$$

Using (3.2) for $s=0$, we have

$$
\begin{aligned}
L(t) \geq & -4(p+2) E(0)+2(p+1)\left(1-\int_{0}^{t} g_{1}(\tau) d \tau\right)\|\nabla u(t)\|_{2}^{2}-\frac{1}{2(p+2)} \int_{0}^{t} g_{1}(\tau)\|\nabla u(t)\|_{2}^{2} d \tau \\
& +2(p+1)\left(1-\int_{0}^{t} g_{2}(\tau) d \tau\right)\|\nabla v(t)\|_{2}^{2}-\frac{1}{2(p+2)} \int_{0}^{t} g_{2}(\tau)\|\nabla v(t)\|_{2}^{2} d \tau \\
& +2(p+1)\left(\int_{0}^{t}\left\|\nabla v_{t}(\tau)\right\|_{2}^{2} d \tau+\int_{0}^{t}\left\|\nabla u_{t}(\tau)\right\|_{2}^{2} d \tau\right)-2(p+1) \beta \\
\geq & 4(p+2)\left(\frac{p+1}{2(p+2)}\left(\left(1-\int_{0}^{t} g_{1}(\tau) d \tau\right)-\frac{1}{4(p+2)(p+1)} \int_{0}^{t} g_{1}(\tau) d \tau\right)\right)\|\nabla u(t)\|_{2}^{2} \\
& +4(p+2)\left(\frac{p+1}{2(p+2)}\left(\left(1-\int_{0}^{t} g_{2}(\tau) d \tau\right)-\frac{1}{4(p+2)(p+1)} \int_{0}^{t} g_{2}(\tau) d \tau\right)\right)\|\nabla v(t)\|_{2}^{2} \\
& -4(p+2) E(0)-2(p+1) \beta \\
\geq & 4(p+2)\left(\frac{p+1}{2(p+2)}\left(k_{1}-\frac{1}{4(p+2)(p+1)} \int_{0}^{t} g_{1}(\tau) d \tau\right)\right)\|\nabla u(t)\|_{2}^{2}-2(p+1) \beta \\
& +4(p+2)\left(\frac{p+1}{2(p+2)}\left(k_{2}-\frac{1}{4(p+2)(p+1)} \int_{0}^{t} g_{2}(\tau) d \tau\right)\right)\|\nabla v(t)\|_{2}^{2}-4(p+2) E(0) \\
\geq & 4(p+2)\left(\frac{p+1}{2(p+2)}\left(\theta_{1}\|\nabla u(t)\|_{2}^{2}+\theta_{2}\|\nabla v(t)\|_{2}^{2}\right)-E(0)-\frac{p+1}{2(p+2)} \beta\right) .
\end{aligned}
$$

Since

$$
\left(\theta_{1}\left\|\nabla u_{0}\right\|_{2}^{2}+\theta_{2}\left\|\nabla v_{0}\right\|_{2}^{2}\right)^{1 / 2}>\alpha_{*} \text { and } E(0)<E_{2} ，
$$

by Lemma 4.2 , there exists a constant $\alpha>\alpha *$ such that

$$
\left(\theta_{1}\|\nabla u(t)\|_{2}^{2}+\theta_{2}\|\nabla v(t)\|_{2}^{2}\right)^{1 / 2} \geq \alpha_{2},
$$

which implies

$$
\frac{p+1}{2(p+2)}\left(\theta_{1}\|\nabla u(t)\|_{2}^{2}+\theta_{2}\|\nabla v(t)\|_{2}^{2}\right) \geq \frac{p+1}{2(p+2)} \alpha_{2}^{2}>E_{2}>E(0) .
$$

Thus, we can let $\beta$ satisfy

$$
(p+1) \beta<2(p+2)\left(E_{2}-E(0)\right)
$$

which implies that there exists $\delta>0$ (independent of $T$ ) such that

$$
L(t) \geq \delta \quad \text { for } t \in[0, T] .
$$

From (4.15) and the definition of $H(t)$, there also exists $\rho>0$ (independent of $T$ ) such that 


$$
H(t) \geq \rho \quad \text { for } t \in[0, T] .
$$

By (4.8), (4.16) and (4.17) it follows that

$$
H(t) H^{\prime \prime}(t)-\frac{p+3}{2} H^{\prime}(t)^{2} \geq \delta \rho \quad \text { for a.e. } t \in[0, T] .
$$

Moreover, we let $s_{0}$ satisfy that

$$
\beta s_{0}+\int_{\Omega} u_{0} u_{1} d x+\int_{\Omega} v_{0} v_{1} d x>0,
$$

which means $H^{\prime}(0)>0$. Thus by $H^{\prime \prime}(t)>0$ we see that $H(t)$ and $H^{\prime}(t)$ is strictly increasing on $[0, T]$.

Setting $y(t)=H(t)^{-(p+1) / 2}$, then we have

$$
\gamma^{\prime}(t)=-\frac{p+1}{2} H(t)^{-(p+3) / 2} H^{\prime}(t)<0,
$$

and

$$
\gamma^{\prime \prime}(t) \leq-\frac{p+1}{2} \delta \rho \gamma(t)^{\frac{p+5}{p+1}}
$$

for all $t \in[0, T]$, which implies that $y(t)$ reaches 0 in finite time, say as $t \rightarrow T^{*}$. Since $T^{*}$ is independent of the initial choice of $T$, we may assume that $T^{*}<T$. This tells us that

$$
\lim _{t \rightarrow T *} H(t)=\infty .
$$

In turn, this implies that

$$
\lim _{t \rightarrow T *}\left(\|\nabla u(t)\|_{2}^{2}+\|\nabla v(t)\|_{2}^{2}\right)=\infty .
$$

Indeed, if

$$
\lim _{t \rightarrow T *}\left(\|u(t)\|_{2}^{2}+\|v(t)\|_{2}^{2}\right)=\infty,
$$

then (4.18) immediately follows. On the contrary, if $\|u(t)\|_{2}^{2}+\|v(t)\|_{2}^{2}$ remains bounded on $\left[0, T^{*}\right)$, then

$$
\lim _{t \rightarrow T *}\left(\int_{0}^{t}\|\nabla u(\tau)\|_{2}^{2} \tau+\int_{0}^{t}\|\nabla v(\tau)\|_{2}^{2} \tau\right)=\infty
$$

so that again (4.18) is satisfied. This implies a contradiction, i.e. $T<\infty$. $\square$

\section{Acknowledgements}

The authors are indebted to the referee for giving some important suggestions which improved the presentations of this article. The study was supported in part by the China NSF Grant No. 10871097, the Qing Lan Project of Jiangsu Province, the Foundation for Young Talents in College of Anhui Province Grant No. 2011SQRL115 and the program sponsored for scientific innovation research of college graduate in Jangsu Province Grant No. 181200000649.

\section{Author details}

${ }^{1}$ Jiangsu Provincial Key Laboratory for Numerical Simulation of Large Scale Complex Systems, School of Mathematical Sciences, Nanjing Normal University, Nanjing 210046, PR China ${ }^{2}$ Department of Mathematics, Anhui Science and Technology University, Feng Yang 233100, Anhui, PR China 
Authors' contributions

$\mathrm{FL}$ and $\mathrm{HGAO}$ carried out all studies in this article.

\section{Competing interests}

The authors declare that they have no competing interests.

\section{Received: 26 April 2011 Accepted: 13 September 2011 Published: 13 September 2011}

\section{References}

1. Cavalcanti, MM, Domingos Cavalcanti, VN, Ferreira, JL: Existence and uniform decay for nonlinear viscoelastic equation with strong damping. Math. Methods Appl. Sci. 24, 1043-1053 (2001). doi:10.1002/mma.250

2. Messaoudi, SA, Tatar, N-E: Global existence and uniform stability of solutions for a quasilinear viscoelastic problem. Math. Methods. Appl. Sci. 30, 665-680 (2007). doi:10.1002/mma.804

3. Renardy, M, Hrusa, WJ, Nohel, JA: Mathematical problems in viscoelasticity. In Pitman Monographs and Surveys in Pure and Applied Mathematics, vol. 35,Wiley, New York (1987)

4. Cavalcanti, MM, Domingos Cavalcanti, VN, Soriano, JA: Exponential decay for the solution of semilinear viscoelastic wave equations with localized damping. Electron. J. Diff. Equ. 44, 1-12 (2002)

5. Cavalcanti, MM, Oquendo, HP: Frictional versus viscoelastic damping in a semilinear wave equation. SIAM J. Control Optim. 42, 1310-1324 (2003). doi:10.1137/\$0363012902408010

6. Berrimi, S, Messaoudi, SA: Exponential decay of solutions to a viscoelastic equation with nonlinear localized damping. Electron. J. Diff. Equ. 88, 1-10 (2004)

7. Messaoudi, SA, Tatar, N-E: Exponential and polynomial decay for a quasilinear viscoelastic problem. Nonlinear Anal. 68 785-793 (2007)

8. Messaoudi, SA: General decay of solutions of a viscoelastic equation. J. Math. Anal. Appl. 341, 1457-1467 (2008) doi:10.1016/j.jmaa.2007.11.048

9. Messaoudi, SA: General decay of the solution energy in a viscoelastic equation with a nonlinear source. Nonlinear Anal. 69, 2589-2598 (2008). doi:10.1016/j.na.2007.08.035

10. Kafini, M, Messaoudi, SA: A blow-up result in a Cauchy viscoelastic problem. Appl. Math. Lett. 21, 549-553 (2008). doi:10.1016/j.aml.2007.07.004

11. Messaoudi, SA: Blow up and global existence in a nonlinear viscoelastic wave equation. Math. Nachr. 260, 58-66 (2003). doi:10.1002/mana.200310104

12. Messaoudi, SA: Blow up of positive-initial-energy solutions of a nonlinear viscoelastic hyperbolic equation. J. Math. Anal. Appl. 320, 902-915 (2006). doi:10.1016/j.jmaa.2005.07.022

13. Song, HT, Zhong, CK: Blow-up of solutions of a nonlinear viscoelastic wave equation. Nonlinear Anal. RWA. 11, 3877-3883 (2010). doi:10.1016/j.nonrwa.2010.02.015

14. Wang, YJ: A global nonexistence theorem for viscoelastic equations with arbitrarily positive initial energy. Appl. Math. Lett. 22, 1394-1400 (2009). doi:10.1016/j.aml.2009.01.052

15. Payne, L, Sattinger, D: Saddle points and instability on nonlinear hyperbolic equations. Isr. Math. J. 22, 273-303 (1975). doi:10.1007/BF02761595

16. Segal, IE: The global Cauchy problem for relativistic scalar field with power interactions. Bull. Soc. Math. France. 91, 129-135 (1963)

17. Medeiros, LA, Miranda, MM: Weak solutions for a system of nonlinear Klein-Gordon equations. Ann. Mat. Pura Appl. 146, 173-183 (1987)

18. Zhang, J: On the standing wave in coupled non-linear Klein-Gordon equations. Math. Methods Appl. Sci. 26, 11-25 (2003). doi:10.1002/mma.340

19. Han, XS, Wang, WM: Global existence and blow-up of solutions for a system of nonlinear viscoelastic wave equations with damping and source. Nonlinear Anal. 71, 5427-5450 (2009). doi:10.1016/..na.2009.04.031

20. Messaoudi, SA, Said-Houari, B: Global nonexistence of positive initial-energy solutions of a system of nonlinear viscoelastic wave equations with damping and source terms. J. Math. Anal. Appl. 365, 277-287 (2010). doi:10.1016/j. jmaa.2009.10.050

21. Liu, WJ: Uniform decay of solutions for a quaslinear system of viscoelastic equations. Nonlinear Anal. 71, 2257-2267 (2009). doi:10.1016/..na.2009.01.060

22. Agre, K, Rammaha, MA: Systems of nonlinear wave equations with damping and source terms. Diff. Integ. Equ. 19, 1235-1270 (2006)

23. Said-Houari, B, Messaoudi, SA, Guesmia, A: General decay of solutions of a nonlinear system of viscoelastic wave equations. Nonlinear Diff. Equ. Appl. (2011)

24. Muñoz Rivera, JE: Global solution on a quasilinear wave equation with memory. Boll. Un. Mat. Ital. B. 8, 289-303 (1994)

25. Muñoz Rivera, JE, Cabanillas Lapa, E: Decay rates of solutions of an anisotropic inhomogeneous n-dimensional viscoelastic equation with polynomially decaying kernels. Commun. Math. Phys. 177, 583-602 (1996). doi:10.1007/ BF02099539

26. Said-Houari, B: Global nonexistence of positive initial-energy solutions of a system of nonlinear wave equations with damping and source terms. Diff. Integ. Equ. 23, 79-92 (2010)

27. Vitillaro, E: Global nonexistence theorems for a class of evolution equations with dissipation. Arch. Ration. Mech. Anal. 149, 155-182 (1999). doi:10.1007/s002050050171

28. Komornik, V: Exact controllability and stabilization. RAM: Research in Applied Mathematics. Masson, Paris. (1994)

doi:10.1186/1687-2770-2011-22

Cite this article as: Liang and Gao: Exponential energy decay and blow-up of solutions for a system of nonlinear viscoelastic wave equations with strong damping. Boundary Value Problems 2011 2011:22. 